\title{
ON THE OPTIMALITY OF VELOCITY AVERAGING LEMMAS
}

\author{
Camillo DE LELLIS ${ }^{a}$, Michael WESTDICKENBERG ${ }^{\text {b,* }}$ \\ ${ }^{a}$ Max-Planck Institute for Mathematics in the Sciences, Inselstr. 22, 04103 Leipzig, Germany \\ ${ }^{\mathrm{b}}$ Institute for Applied Mathematics, University of Bonn, Wegelerstr. 10, 53115 Bonn, Germany
}

Received 13 January 2003, accepted 25 February 2003

\begin{abstract}
Studying weak solutions of Burgers' equation with finite entropy dissipation we show the sharpness of recent results of Jabin and Perthame on velocity averaging. Similar arguments give bounds on the regularity of asymptotic finite-energy states for some variational problems of Ginzburg-Landau type.

(C) 2003 L'Association Publications de l'Institut Henri Poincaré. Published by Elsevier B.V. All rights reserved
\end{abstract}

RÉSUMÉ. - Nous construisons des solutions faibles de l'équation de Burgers à dissipation finie de l'entropie et montrons que les exposants de régularité obtenus récemment par Jabin et Perthame pour les théorèmes de moyennes en vitesse sont optimal. Nous étudions aussi la régularité des états asymptotiques d'un problème variationnel de type Ginzburg-Landau.

(C) 2003 L'Association Publications de l'Institut Henri Poincaré. Published by Elsevier B.V. All rights reserved

MSC: 35L65; 35B65; 46E35

Keywords: Kinetic equations; Velocity averaging; Regularity

\section{Introduction}

It is well-known that entropy solutions of Burgers' equation

$$
\partial_{t} u+\frac{1}{2} \partial_{x} u^{2}=0 \quad \text { on } \Omega:=(0,1) \times \mathbf{R}
$$

are locally in $B V$, see [9]. This is no longer true for a general weak solution, which can have oscillations. Thus an improved regularity can only be expected if additional assumptions are imposed. We will consider here the following class of functions

DEFINITION 1.1. - Let $\mathcal{S}$ be the set of functions $u \in L^{\infty}(\Omega)$ with

- $u$ is a weak solution of (1),

\footnotetext{
* Corresponding author.

E-mail addresses: delellis@mis.mpg.de (C. De Lellis), mwest@iam.uni-bonn.de (M. Westdickenberg).
} 
- the entropy dissipation $-\mu_{\eta}:=\partial_{t} \eta(u)+\partial_{x} q(u)$ is a Radon measure for every convex entropy-entropy flux pair $(\eta, q)$.

We stress that it is quite natural to consider this class of functions. First, $\mathcal{S}$ contains all entropy solutions. For these, $\mu_{\eta}$ is non-negative. Second, for $u \in \mathcal{S}$ the conservation law allows a kinetic formulation, see [5]. Once we are given a kinetic formulation, we can apply velocity averaging arguments to study the regularity of weak solutions $u$. Using this remark we will show the sharpness of some recent results contained in [6].

To explain this point we recall the kinetic formulation for scalar conservation laws, first introduced in [8]. Assume $u \in L^{\infty}\left(\mathbf{R}^{+} \times \mathbf{R}^{n}\right)$ is an entropy solution of some scalar conservation law $\partial_{t} u+\operatorname{div}_{x} A(u)=0$ in several space dimensions. One introduces the local Maxwellian

$$
\chi(v, u)= \begin{cases}1 & \text { for } 0<v \leqslant u \\ -1 & \text { for } u \leqslant v<0 \\ 0 & \text { otherwise }\end{cases}
$$

Then there exists a non-negative Radon measure $m$ in $(t, x) \in \mathbf{R}^{+} \times \mathbf{R}^{n}$ and $v \in \mathbf{R}$ such that $\chi(v, u(t, x))$ satisfies the transport equation

$$
\partial_{t} \chi(v, u)+A^{\prime}(v) \cdot \nabla_{x} \chi(v, u)=\partial_{v} m \quad \text { in } \mathcal{D}^{\prime}
$$

Conversely, if there exist a bounded function $u$ and a non-negative measure $m$ such that (2) holds, then $u$ is the unique entropy solution of the conservation law. One can show that even if the entropy dissipation of $u$ is not non-negative but a Radon measure only, then still $\chi(v, u)$ satisfies a transport equation (2), see [5]. In that case, however, the measure $m$ changes sign in general.

Since $u=\int \chi(v, u) d v$, velocity averaging lemmas can be applied to (2). Under certain assumptions on the non-degeneracy of the flux $A$, they yield regularity results for $u$, see $[8,6]$. We stress the fact that the velocity averaging technique as it is currently available does not use the non-negativity of $m$ : one obtains exactly the same regularity if $m$ in (2) is a Radon measure only.

For functions $u \in \mathcal{S}$ we obtain the transport equation

$$
\partial_{t} \chi(v, u)+v \partial_{x} \chi(v, u)=\partial_{v} m \quad \text { in } \mathcal{D}^{\prime}
$$

for some Radon measure $m$. Then the results in [6] yield $u \in W_{\mathrm{loc}}^{\alpha, 3 / 2}$ for all $\alpha<\frac{1}{3}$. We prove below that this gain of regularity is essentially sharp.

Let us fix some notation. For numbers $\alpha \in \mathbf{R}$ and $1 \leqslant p, q \leqslant \infty$ we denote by $B_{p, q}^{\alpha}$ the usual Besov space (see [10, Section 10]).

DEFINITION 1.2. - Let $\sigma \geqslant 1$ and consider the one-parameter family of spaces

$$
\left\{B_{r, r}^{1 / \sigma} \text { for } 1 \leqslant r<\sigma, B_{r, \infty}^{1 / r} \text { for } r \geqslant \sigma\right\} .
$$


We say that a set of functions $\mathcal{S} \subset L^{\infty}(\Omega)$ is not better than (4) locally if

$$
\text { for every triple }(\alpha, p, q) \text { with } \begin{cases}\alpha>1 / \max \{\sigma, p\} & \\ \operatorname{or} \alpha=1 / \sigma, & p<\sigma, q<p, \\ \operatorname{or} \alpha=1 / p, & p \geqslant \sigma, q<\infty,\end{cases}
$$

there exist $u \in \mathcal{S}$ and a test function $\psi \in \mathcal{D}(\Omega)$ such that $\psi u \notin B_{p, q}^{\alpha}$.

We can prove the following result

Proposition 1.3. - Let $\mathcal{S}$ be the set of Definition 1.1. Then we have

$\mathcal{S}$ is not better than (4) locally with $\sigma=3$.

Thanks to the embedding $W^{\alpha, p} \subset B_{p, q}^{\alpha}$ for $q \geqslant 2$ (see [10, Section 11.4]) we conclude that $u \notin W_{\mathrm{loc}}^{\alpha, p}$ if $\alpha>1 / 3$, or if $\alpha \leqslant 1 / 3$ and $p \geqslant 1 / \alpha$. Thus the results in [6] are essentially sharp in the number of derivatives, whereas the integrability could possibly be improved somewhat.

We prove Proposition 1.3 by constructing weak solutions of (1) with low regularity. The argument can be generalized to more general fluxes. It is also possible to derive upper bounds for the regularity of entropy solutions. We refer to Section 3 for further discussion.

We mention that the starting point of our investigation was a class of variational problems of Ginzburg-Landau type, see [2,7]. Here one considers a family of functionals $F_{\varepsilon}$ defined on curl free planar vector fields (see (15)), and tries to characterize possible limits $w$ of sequences $\left\{w_{\varepsilon}\right\}_{\varepsilon}$ with $\lim \sup _{\varepsilon} F_{\varepsilon}\left(w_{\varepsilon}\right)<\infty$. Combining an example of [1] with a construction of [4] one can show that there exist limits which do not belong to $B V$. On the other hand, the results in [6] can again be applied and give $w \in W^{\alpha, 3 / 2}$ for all $\alpha<1 / 3$. Using estimates similar to those in Section 2 we can show that generically $w$ is not better than (4) with $\sigma=3$, see Proposition 3.3.

\section{Proof of Proposition 1.3}

We proceed in three steps.

Step 1. A family of weak solutions of (1). Assume that $\left\{\Delta_{k}\right\}_{k} \in \ell^{1}$ and $\left\{c_{k}\right\}_{k} \in \ell^{3}$ are two nonincreasing sequences of positive numbers which will be determined later on. Let

$$
x_{1}^{-}:=\sum_{k=1}^{\infty} \Delta_{k}
$$

Inductively, we now define numbers

$$
x_{k+1}^{-}:=x_{k}^{-}-\Delta_{k} \quad \text { and } \quad x_{k}^{+}:=x_{k}^{-}-\frac{\Delta_{k}}{2}
$$


and intervals $I_{k}:=\left[x_{k}^{+}, x_{k}^{-}\right]$for $k \in \mathbf{N}$. We prescribe initial data

$$
u(0, \cdot)=\sum_{k=1}^{\infty} c_{k} \mathbf{1}_{I_{k}}
$$

and construct a weak solution $u$ of (1) with (5), consisting of shocks only. For a jump connecting 0 and $c_{k}$, the Rankine-Hugoniot condition gives a shock speed $s_{k}:=\frac{1}{2} c_{k}$. Define vectors $X_{k}:=\left(1, s_{k}\right)$. Then

$$
u=\sum_{k=1}^{\infty} c_{k} \mathbf{1}_{A_{k}} \quad \text { where } A_{k}:=\left(\{0\} \times I_{k}\right)+\mathbf{R}^{+} X_{k} .
$$

This $u$ is a weak solution of (1), but it is entropy violating. We compute $\partial_{t} \chi(v, u)+$ $v \partial_{x} \chi(v, u)$ and show that it is the $v$-derivative of a Radon measure $m \in \mathcal{M}_{\text {loc }}\left(\mathbf{R}_{v} \times\right.$ $\left.\mathbf{R}_{t}^{+} \times \mathbf{R}_{x}\right)$. We restrict our attention on the set $\Omega:=(0,1)_{t} \times \mathbf{R}_{x}$. Define segments

$$
J_{k}^{ \pm}:=\left(\{0\} \times x_{k}^{ \pm}\right)+[0,1] X_{k} \text { for } j \in \mathbf{N} .
$$

The unit normal to $J_{k}$ is given by $\left(1+s_{k}^{2}\right)^{-1 / 2}\left(s_{k},-1\right)$. Then we obtain

$$
\partial_{t} \chi(v, u)+v \partial_{x} \chi(v, u)=-\sum_{k=1}^{\infty} h_{k}(v)\left(\mathcal { H } ^ { 1 } \left\llcornerJ_{k}^{+}-\mathcal{H}^{1}\left\llcorner J_{k}^{-}\right) \quad \text { in } \mathcal{D}^{\prime}(\Omega)\right.\right.
$$

for every $v \in \mathbf{R}$, where the function $h_{k}$ is defined as

$$
h_{k}(v):=(1, v) \cdot \frac{\left(s_{k},-1\right)}{\sqrt{1+s_{k}^{2}}}=\frac{c_{k} / 2-v}{\sqrt{1+c_{k}^{2} / 4}} \text { for } v \in\left(0, c_{k}\right]
$$

and $h_{k}(v)=0$ otherwise. We can define the measure $m$ as

$$
m:=-\sum_{k=1}^{\infty} H_{k}(v)\left(\mathcal { H } ^ { 1 } \left\llcornerJ_{k}^{+}-\mathcal{H}^{1}\left\llcorner J_{k}^{-}\right),\right.\right.
$$

where $H_{k}$ is a $v$-primitive of (6). For example we can choose

$$
H_{k}(v):=\int_{0}^{v} h_{k}(s) d s= \begin{cases}\frac{1}{2} \frac{c_{k} v-v^{2}}{\sqrt{1+c_{k}^{2} / 4}} & \text { for } v \in\left[0, c_{k}\right], \\ 0 & \text { otherwise. }\end{cases}
$$

The function $H_{k}$ is non-negative. Its $L^{1}(\mathbf{R})$-norm equals

$$
\int_{\mathbf{R}} H_{k}(v) d v=\frac{1}{12} \frac{c_{k}^{3}}{\sqrt{1+c_{k}^{2} / 4}}
$$


C. DE LELLIS, M. WESTDICKENBERG / Ann. I. H. Poincaré - AN 20 (2003) 1075-1085 1079

i.e., is essentially cubic in the shock strength. Then we have

$$
\|m\|_{\mathcal{M}(\mathbf{R} \times \Omega)}=\sum_{k=1}^{\infty} 2 \cdot \sqrt{1+\frac{c_{k}^{2}}{4}} \cdot \frac{1}{12} \frac{c_{k}^{3}}{\sqrt{1+c_{k}^{2} / 4}}=\frac{1}{6} \sum_{k=1}^{\infty} c_{k}^{3} .
$$

Since $\left\{c_{k}\right\}_{k} \in \ell^{3}$ by assumption, $m$ is a finite measure.

Step 2. Besov norms and main estimate. We will work with Besov spaces $B_{p, q}^{\alpha}$ defined on $\mathbf{R}^{2}$. Several equivalent norms are known for these spaces. A particularly useful one is the "local means" norm. Let $\Phi, \varphi \in \mathcal{D}$ be mollifiers and assume that $\varphi$ satisfies a certain moment condition (we refer to [10, Section 11 for details]). Put $\varphi_{s}(t, x):=s^{-2} \varphi(t / s, x / s)$ for $(t, x) \in \mathbf{R}^{2}$ and $s>0$. Then

$$
\|u\|_{B_{p, q}^{\alpha}} \approx\|u \star \Phi\|_{L^{p}}+\left(\int_{0}^{\varepsilon} s^{-\alpha q}\left\|u \star \varphi_{s}\right\|_{L^{p}}^{q} \frac{d s}{s}\right)^{1 / q} \quad \text { if } q<\infty
$$

for some $\varepsilon>0$. If $q=\infty$ the integral must be replaced by a sup. We also use

$$
\|u\|_{B_{p, q}^{\alpha}} \approx\|u\|_{B_{p, q}^{\alpha-1}}+\left\|\partial_{t} u\right\|_{B_{p, q}^{\alpha-1}}+\left\|\partial_{x} u\right\|_{B_{p, q}^{\alpha-q}},
$$

and will concentrate on the $\partial_{x} u$-part with $\alpha<1$. For negative regularity the function $\varphi$ may be a standard mollifier (satisfying no moment conditions). Moreover, the first term in (9) can be dropped, see [11, Remark 6]. Thus we only have to consider

$$
\left\|\partial_{x} u\right\|_{B_{p, q}^{\alpha-1}} \approx\left(\int_{0}^{\varepsilon} s^{(1-\alpha) q}\left\|\left(\partial_{x} u\right) \star \varphi_{s}\right\|_{L^{p}}^{q} \frac{d s}{s}\right)^{1 / q} \quad \text { if } q<\infty
$$

with the obvious modification for $q=\infty$. We remark in passing that

$$
\|D u\|_{\mathcal{M}} \approx \sup _{0<s<\varepsilon}\left\|D u \star \varphi_{s}\right\|_{L^{1}} \quad \text { for all } u \in B V .
$$

This follows easily from the lower semi-continuity of the variation. Hence the space $B V$ fits very neatly into the Besov space framework. (In fact, in his first work on Caccioppoli sets [3] De Giorgi defined the space $B V$ as the set of all $u \in L^{\infty}$ such that (10) stays finite, where $\varphi_{s}$ is the heat kernel.)

To fix ideas, we let $\varphi$ be a non-negative, radially symmetric test function with

$$
\varphi(t, x)= \begin{cases}1 & \text { for }(t, x) \in B_{1}(0), \\ 0 & \text { for }(t, x) \in \mathbf{R}^{2} \backslash B_{2}(0) .\end{cases}
$$

We now fix some test function $\psi \in \mathcal{D}(\Omega)$ which equals 1 on a sufficiently large open set, say $[1 / 4,3 / 4] \times[-1,3]$. Note that $\partial_{x}(\psi u)=u \partial_{x} \psi+\psi \partial_{x} u$. Since $u \partial_{x} \psi \in L^{\infty}$, clearly 
$u \partial_{x} \psi \in B_{p, q}^{\alpha-1}$ for any $\alpha<1$. Thus to show that $\partial_{x}(\psi u) \notin B_{p, q}^{\alpha-1}$ for some $\alpha<1$, it is sufficient to show that $\psi \partial_{x} u \notin B_{p, q}^{\alpha-1}$.

We proceed as follows: the partial derivative of $u$ is a measure

$$
\begin{aligned}
\partial_{x} u & =\sum_{k=1}^{\infty} \frac{c_{k}}{\sqrt{1+c_{k}^{2} / 4}}\left(\mathcal { H } ^ { 1 } \left\llcornerJ_{k}^{+}-\mathcal{H}^{1}\left\llcorner J_{k}^{-}\right)\right.\right. \\
& =: \sum_{k=1}^{\infty}\left(\mu_{k}^{+}-\mu_{k}^{-}\right) .
\end{aligned}
$$

By construction, the distance between $J_{k}^{ \pm}$and its neighbors is not less than $\Delta_{k} /(2(1+$ $\left.s_{k}^{2}\right)^{1 / 2}$ ). If we choose $c_{k} \leqslant 2$, then this distance is bigger than $\Delta_{k} / 4$. Hence, for all $k$ with $4 s<\Delta_{k} / 4$, only one line $J_{k}^{ \pm}$contributes to the convolution $\left(\partial_{x} u\right) \star \varphi_{s}$ (since $\varphi_{s}$ is supported in a ball of radius $2 s$ ). Thus

$$
\left|\left(\partial_{x} u\right) \star \varphi_{s}\right|(t, x) \geqslant \sum_{4 s<\Delta_{k} / 4}\left|\mu_{k}^{ \pm} \star \varphi_{s}\right|(t, x),
$$

where the functions appearing in the sum have pairwise disjoint supports. Since $\varphi_{s}$ is supported in a ball of radius $2 s$ and $\psi$ is 1 on $[1 / 4,3 / 4] \times[-1,3]$

$$
\left|\left(\partial_{x} u\right) \star \varphi_{s}\right|(t, x)=\left|\left(\psi \partial_{x} u\right) \star \varphi_{s}\right|(t, x) \quad \text { for } t \in[1 / 4+2 s, 3 / 4-2 s] .
$$

Moreover, since $\varphi$ is non-negative, each summand of (11) can be estimated from below

$$
\left|\mu_{k}^{ \pm} \star \varphi_{s}\right|(t, x) \geqslant \begin{cases}2 c_{k} s^{-2} \frac{\sqrt{s^{2}-y^{2}}}{\sqrt{1+c_{k}^{2} / 4}} & \text { for } y \in[-s, s], \\ 0 & \text { otherwise, }\end{cases}
$$

where $y:=\left(x-\left(x_{k}^{ \pm}+t c_{k} / 2\right)\right) / \sqrt{1+c_{k}^{2} / 4}$ for $x \in \mathbf{R}$ and $t \in[s, 1-s]$, see Fig. 1 . These remarks allow us to give the following estimate:

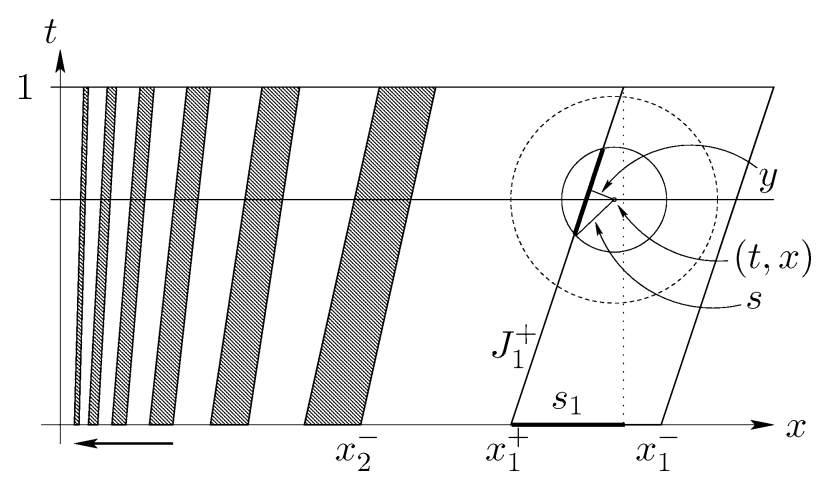

Fig. 1. Local means $\left(\partial_{x} u\right) \star \varphi_{s}$. 
C. DE LELLIS, M. WESTDICKENBERG / Ann. I. H. Poincaré - AN 20 (2003) 1075-1085 1081

$$
\begin{aligned}
& \int_{0}^{1} \int_{0}^{2 x_{1}^{-}}\left|\left(\psi \partial_{x} u\right) \star \varphi_{s}\right|^{p}(t, x) d x d t \\
& \quad \geqslant \sum_{4 s<\Delta_{k} / 4} 2^{p} c_{k}^{p}\left(1+\frac{c_{k}^{2}}{4}\right)^{(1-p) / 2} s^{1-p} \int_{1 / 4+2 s}^{3 / 4-2 s} \int_{-1}^{1}\left(1-z^{2}\right)^{p / 2} d z d t \\
& \geqslant c s^{1-p} \sum_{4 s<\Delta_{k} / 4} c_{k}^{p} \quad \text { for some constant } c=c(p, \varepsilon) .
\end{aligned}
$$

Then we have

$$
\left\|\left(\psi \partial_{x} u\right) \star \varphi_{s}\right\|_{L^{p}} \geqslant c s^{1 / p-1}\left(\sum_{4 s<\Delta_{k} / 4} c_{k}^{p}\right)^{1 / p}
$$

Step 3. Conclusion. Now we consider three cases:

Case I: $p \geqslant 3$. If $\alpha>1 / p$ then $u \notin B_{p, q}^{\alpha}$. If $q<\infty$ then $u \notin B_{p, q}^{1 / p}$.

Since $\ell^{3} \hookrightarrow \ell^{p}$ if $p \geqslant 3$, we have $\sum_{4 s<\Delta_{k} / 4} c_{k}^{p}=\mathcal{O}(1)$ as $s \downarrow 0$. Therefore, if $q<\infty$ and $\alpha \geqslant 1 / p,(12)$ yields

$$
\left\|\psi \partial_{x} u\right\|_{B_{p, q}^{\alpha-1}} \geqslant c\left(\int_{0}^{\varepsilon} s^{(1-\alpha) q} s^{(1 / p-1) q} \frac{d s}{s}\right)^{1 / q}=c\left(\int_{0}^{\varepsilon} \frac{d s}{s^{1+(\alpha-1 / p) q}}\right)^{1 / q}=\infty .
$$

Similarly, if $q=\infty$ and $\alpha>1 / p$, then

$$
\left\|\psi \partial_{x} u\right\|_{B_{p, \infty}^{\alpha-1}} \geqslant c \sup _{0<s<\varepsilon} s^{1-\alpha} s^{1 / p-1}=\infty .
$$

This proves that $u$ cannot be better than $B_{p, \infty}^{1 / p}$ locally for $p \geqslant 3$.

Case II: $p<3, \alpha>1 / 3$. Then $u \notin B_{p, q}^{\alpha}$.

We consider sequences of the form

$$
\Delta_{k}=k^{-\beta} \quad \text { and } \quad c_{k}=k^{-\gamma} \quad \text { for } k \in \mathbf{N}
$$

with suitable numbers $\beta, \gamma$. Since we assume that $\left\{\Delta_{k}\right\}_{k} \in \ell^{1}$ and $\left\{c_{k}\right\}_{k} \in \ell^{3}$, we require $\beta>1$ and $\gamma>1 / 3$. Then we have

$$
4 s<\frac{\Delta_{k}}{2} \Longleftrightarrow k<(16 s)^{-1 / \beta} .
$$

As a consequence, we obtain the following estimate:

$$
\text { if } \gamma<1 / p \text {, then } \sum_{4 s<\Delta_{k} / 4} c_{k}^{p}=\mathcal{O}\left(s^{-(1-\gamma p) / \beta}\right) \quad \text { as } s \downarrow 0 \text {. }
$$

Since $\beta>1$ and $\gamma>1 / 3$, we have

$$
0<\frac{1-\gamma p}{\beta}<1-\gamma p<1-\frac{p}{3}=\frac{3-p}{3},
$$


and thus

$$
\frac{1}{p}>\frac{1}{p}\left[1-\frac{1-\gamma p}{\beta}\right]>\frac{1}{3}
$$

Assume now that $\alpha>1 / 3$. Then we can find $\beta>1$ and $1 / p>\gamma>1 / 3$ with

$$
-\delta:=(1-\alpha)+\left(\frac{1}{p}-1\right)-\frac{1}{p} \frac{1-\gamma p}{\beta}<0,
$$

and therefore (12) yields

$$
\left\|\psi \partial_{x} u\right\|_{B_{p, q}^{\alpha-1}} \geqslant c\left(\int_{0}^{\varepsilon} s^{(1-\alpha) q} s^{(1 / p-1) q} s^{-q(1-\gamma p) / p \beta} \frac{d s}{s}\right)^{1 / q}=c\left(\int_{0}^{\varepsilon} s^{-\delta q} \frac{d s}{s}\right)^{1 / q}=\infty .
$$

A similar estimate holds in case $q=\infty$.

Case III: $q<p<3$. Then $u \notin B_{p, q}^{1 / 3}$.

To achieve this, we need a more refined estimate. Let $\varphi(z):=z(\log z)^{\sigma}$ for some $\sigma>1$. This $\varphi$ is strictly monotone increasing for $z \geqslant 1$ and unbounded. Hence, $\varphi$ is invertible and the inverse function $\varphi^{-1}$ is increasing and unbounded, too. We define

$$
\Delta_{k}:=(\varphi(k+2))^{-1} \quad \text { and } \quad c_{k}:=(\varphi(k+2))^{-1 / 3} \text { for } k \in \mathbf{N} .
$$

Then $\left\{\Delta_{k}\right\}_{k} \in \ell^{1}$ and $\left\{c_{k}\right\}_{k} \in \ell^{3}$ as needed. We estimate

$$
\sum_{4 s<\Delta_{k} / 4} c_{k}^{p} \geqslant \int_{1}^{\varphi^{-1}(1 /(16 s))-2}(\varphi(z+2))^{-p / 3} d z=\int_{\varphi(3)}^{1 /(16 s)} y^{-p / 3} \frac{d y}{\varphi^{\prime}\left(\varphi^{-1}(y)\right)}
$$

with a substitution of variables $y(z):=\varphi(z+2)$. Now we claim that

$$
\varphi^{\prime}\left(\varphi^{-1}(y)\right) \leqslant c(\log y)^{\sigma} \text { for } y \text { large, }
$$

with $c>0$ some constant. If (13) holds we can estimate

$$
\begin{aligned}
\sum_{4 s<\Delta_{k} / 4} c_{k}^{p} & \geqslant c(-\log 16 s)^{-\sigma} \int_{\varphi(3)}^{1 /(16 s)} y^{-p / 3} d y \\
& \geqslant c(-\log 16 s)^{-\sigma} s^{-(3-p) / 3} \text { for } 0<s<\varepsilon
\end{aligned}
$$

for suitable $\varepsilon$ and $c=c(\varepsilon, \sigma)$. Since $q<p$ we can choose $\sigma>1$ small enough such that $\sigma q / p \leqslant 1$. Then (12) yields

$$
\left\|\psi \partial_{x} u\right\|_{B_{p, q}^{-2 / 3}} \geqslant c\left(\int_{0}^{\varepsilon}(-\log 16 s)^{-\sigma q / p} \frac{d s}{s}\right)^{1 / q}=\infty .
$$

This proves that $u$ does not belong to $B_{p, q}^{1 / 3}$ locally. 
C. DE LELLIS, M. WESTDICKENBERG / Ann. I. H. Poincaré - AN 20 (2003) 1075-1085 1083

It remains to show (13). Since $\varphi^{-1}$ is increasing and unbounded, (13) is equivalent to

$$
\varphi^{\prime}(z) \leqslant c(\log \varphi(z))^{\sigma} \text { for } z \text { large. }
$$

To establish this, we compute

$$
\begin{aligned}
\varphi^{\prime}(z) & =(\log z)^{\sigma}\left[1+\sigma(\log z)^{-1}\right] \\
& \leqslant c(\log z)^{\sigma} \leqslant c\left(\log \left(z(\log z)^{\sigma}\right)\right)^{\sigma}=c(\log \varphi(z))^{\sigma}
\end{aligned}
$$

for some $c>0$ and $z$ large. This proves our claim.

\section{Concluding remarks}

\subsection{Weak solutions}

If we have better regularity in the right-hand side of (3), then $u$ can be smoother. Refining the calculations of Section 2 we can prove

PROPOSITION 3.1. - Let $\sigma \in[1,3]$, and consider the family $\mathcal{S}$ of bounded weak solutions of (1) for which the distribution

$$
\partial_{v}^{2-\sigma}\left\{\partial_{t} \chi(v, u)+v \partial_{x} \chi(v, u)\right\} \text { is a Radon measure. }
$$

Then $\mathcal{S}$ is not better than (4) locally.

Assume for example that $\partial_{v} m$ in (3) itself is a measure (hence $\sigma=2$ ). Then, instead of estimating the norm of $H_{k}$ in (7) and (8), we consider

$$
\left\|\partial_{v} m\right\|_{\mathcal{M}(\mathbf{R} \times \Omega)}=\sum_{k=1}^{\infty} 2 \cdot \sqrt{1+c_{k}^{2} / 4} \cdot \int_{\mathbf{R}}\left|h_{k}(v)\right| d v=\frac{1}{2} \sum_{k=1}^{\infty} c_{k}^{2} .
$$

Then the arguments in Section 2 can be repeated in a straightforward way and they give the result above, i.e., that $u$ can have up to $1 / 2$ derivatives. When even $\partial_{v}^{2} m$ is a measure, then $u$ can have a full derivative. We conjecture that in this case $u$ is in $B V$ locally.

Our construction can be adapted for different fluxes.

PROPOSITION 3.2. - Let $\sigma \geqslant 3$, and consider the family $\mathcal{S}$ of bounded weak solutions of $\partial_{t} u+\frac{1}{\sigma-1} \partial_{x} u^{\sigma-1}=0$ on $(0,1) \times \mathbf{R}$ such that

$$
\partial_{t} \chi(v, u)+v^{\sigma-2} \partial_{x} \chi(v, u)=\partial_{v} m \text { in } \mathcal{D}^{\prime},
$$

for some Radon measure $m$. Then $\mathcal{S}$ is not better than (4) locally.

This suggests that the regularity of $u$ depends on the non-degeneracy of the flux. That is what we expect from the velocity averaging arguments, see [8].

We conclude by giving an exact statement of what examples can be constructed for the variational problems cited in the introduction. Let $\Omega \subset \mathbf{R}^{2}$ be bounded and define

$$
F_{\varepsilon}(w):=\int_{\Omega} \frac{\left(1-|w|^{2}\right)^{2}}{\varepsilon}+\varepsilon|\nabla w|^{2} \quad \text { for } w \in H^{1}\left(\Omega, \mathbf{R}^{2}\right) .
$$


1084 C. DE LELLIS, M. WESTDICKENBERG / Ann. I. H. Poincaré - AN 20 (2003) 1075-1085

Proposition 3.3. - There exists $\left\{w_{\varepsilon}\right\}_{\varepsilon \downarrow 0} \subset L^{\infty}\left(\Omega, \mathbf{R}^{2}\right)$ such that

- $\nabla \times w_{\varepsilon}=0$ and $\lim \sup _{\varepsilon} F_{\varepsilon}\left(w_{\varepsilon}\right)<\infty$;

- $w_{\varepsilon}$ converges (strongly in $L^{p}$ for every $p<\infty$ ) to a $w$ which locally is not better than (4) with $\sigma=3$.

\subsection{Entropy solutions}

Similar arguments can also be applied for entropy solutions. Let us consider the flux $f(u):=\frac{1}{\sigma+1} u^{\sigma+1}$ for $\sigma \geqslant 1$. Since $f$ is convex for $u$ non-negative, only decreasing jumps satisfy the entropy condition. Increasing jumps must be replaced by rarefaction waves.

We want to use a construction as in Step 1 of Section 2, i.e., we define suitable piecewise constant initial data. We start with sequences $\left\{\Delta_{k}\right\}_{k} \in \ell^{1}$ and $\left\{c_{k}\right\}_{k}$ of positive numbers, and put inductively

$$
x_{1}^{+}:=\sum_{k=1}^{\infty} \Delta_{k} \quad \text { and } \quad x_{k+1}^{+}:=x_{k}^{+}-\Delta_{k+1}
$$

for $k \in \mathbf{N}$. An initial jump connecting 0 to $c_{k}$ evolves into a rarefaction wave whose leading edge moves with speed $s_{k}^{\prime}:=f^{\prime}\left(c_{k}\right)=c_{k}^{\sigma}$. We want to ensure that waves do not interact in the time interval $[0,1]$. Then $\Delta_{k}$ and $c_{k}$ cannot be chosen independently. Instead, we are forced to assume $s_{k}^{\prime} \leqslant \Delta_{k}$ for $k \in \mathbf{N}$, i.e., $\left\{c_{k}\right\}_{k} \in \ell^{\sigma}$. We emphasize that $\left\{c_{k}\right\}_{k} \in \ell^{\sigma+2}$ would be sufficient to make $m$ in the kinetic equation (16) a bounded measure. But since rarefaction waves need more space, better summability of $\left\{c_{k}\right\}_{k}$ is required. To have the densest possible packing of shocks, we put $\Delta_{k}:=c_{k}^{\sigma}$. We define

$$
x_{k+1}^{-}:=x_{k}^{+}-s_{k+1} \quad \text { with } s_{k}:=\frac{f\left(c_{k}\right)-f(0)}{c_{k}-0}=\frac{1}{\sigma+1} c_{k}^{\sigma}
$$

for $k \in \mathbf{N}$. Then the shock starting at $x_{k}^{-}$and connecting $c_{k}$ to 0 hits the rarefaction wave at time $t=1$, see Fig. 2. As in Section 2 we can write down a kinetic equation (16). We observe that only the jumps contribute to the non-negative measure $m$ which again is bounded. We note that for all $k$ with $\sqrt{\left|l_{k}\right|^{2}-\left|l_{k}^{\prime}\right|^{2}} \geqslant 4 s$, only one jump (and no

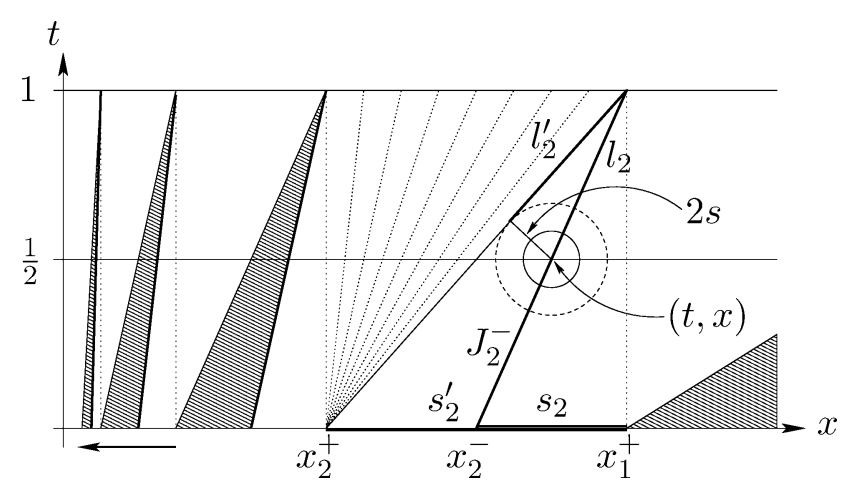

Fig. 2. Entropy solutions. 
C. DE LELLIS, M. WESTDICKENBERG / Ann. I. H. Poincaré - AN 20 (2003) 1075-1085 1085

rarefaction wave) is involved in the convolution $\left(\partial_{x} u\right) \star \varphi_{s}(t, x)$ if $t \leqslant \frac{1}{2}$, see Fig. 2 . This amounts to

$$
16 s \leqslant \frac{\sigma}{\sigma+1} \frac{c_{k}^{\sigma}}{\sqrt{1+c_{k}^{2 \sigma}}}=\frac{\sigma}{\sigma+1} \frac{\Delta_{k}}{\sqrt{1+\Delta_{k}^{2}}} .
$$

Arguing as in Step 3 of Section 2, we can then prove

Proposition 3.4. - Let $\sigma \geqslant 1$, and consider the family $\mathcal{S}$ of bounded entropy solutions of $\partial_{t} u+\frac{1}{\sigma+1} \partial_{x} u^{\sigma+1}=0$ on $(0,1) \times \mathbf{R}$ such that

$$
\partial_{t} \chi(v, u)+v^{\sigma} \partial_{x} \chi(v, u)=\partial_{v} m \quad \text { in } \mathcal{D}^{\prime},
$$

for some Radon measure $m$. Then $\mathcal{S}$ is not better than (4) locally.

This corresponds to the regularity conjectured in [8].

\section{Acknowledgements}

Camillo De Lellis acknowledges partial support by the EU Network Hyperbolic and kinetic equations HPRN-CT-2002-00282. Michael Westdickenberg acknowledges partial support by the Deutsche Forschungsgemeinschaft through SFB 611 Singularities and scaling in mathematical models of the University of Bonn.

\section{REFERENCES}

[1] L. Ambrosio, C. De Lellis, C. Mantegazza, Line energies for gradient vector fields in the plane, Calc. Var. Partial Differential Equations 9 (4) (1999) 327-355.

[2] P. Aviles, Y. Giga, A mathematical problem related to the physical theory of liquid crystal configurations, Proc. Centre Math. Anal. Austral. Nat. Univ. 12 (1987) 1-16.

[3] E. De Giorgi, Definizione ed espressione analitica del perimetro di un insieme, Atti Accad. Naz. Lincei. Rend. Cl. Sci. Fis. Mat. Nat. 14 (8) (1953) 390-393.

[4] C. De Lellis, Energie di linea per campi di gradienti, Ba. D. Thesis, University of Pisa, 1999.

[5] C. De Lellis, F. Otto, M. Westdickenberg, Structure of entropy solutions for multidimensional scalar conservation laws, Preprint $n^{\circ}$ 95/2002 at http://www.mis.mpg.de/ preprints/2002.

[6] P.-E. Jabin, B. Perthame, Regularity in kinetic formulations via averaging lemmas. A tribute to J.-L. Lions, ESAIM Control Optim. Calc. Var. 8 (2002) 761-774.

[7] W. Jin, R.V. Kohn, Singular perturbation and the energy of folds, J. Nonlinear Sci. 10 (3) (2000) 355-390.

[8] P.-L. Lions, B. Perthame, E. Tadmor, A kinetic formulation of multidimensional scalar conservation laws and related equations, J. Amer. Math. Soc. 7 (1994) 169-191.

[9] O.A. Oleinik, Discontinuous solutions of nonlinear differential equations, Transl. Amer. Math. Soc. Ser. 226 (1957) 95-172.

[10] H. Triebel, Fractals and Spectra Related to Fourier Analysis and Function Spaces, in: Monographs Math., Vol. 91, Birkhäuser, Basel, 1997.

[11] H. Triebel, H. Winkelvoss, A Fourier analytical characterization of the Hausdorff dimension of a closed set and of related Lebesgue spaces, Studia Math. 121 (2) (1996) 149-166. 